\title{
Review of: "A Step Towards Environmental Mitigation: How Do Economic Complexity And Natural Resources Matter? Focusing On Different Institutional Quality Level Countries"
}

yan tan

Potential competing interests: The author(s) declared that no potential competing interests exist.

1. The introduction is well written and puts their paper in context, especially its main contribution to the existing literature in this field. The authors do a good job summarizing the previous literature. However, the first paragraph states: "Accordingly, approximately $68 \%$ of greenhouse gases emitted into the atmosphere....". There is no citation for it. The introduction is a bit long; for instance, figure three and its related texts are excess.

2. About the literature review, this subsection is adequate and contextualized in a good way the researched subject. In general, the author does a good job summarizing the previous literature. Moreover, the literature review is well written and clearly outlines the diversity of opinions about the problem. The authors include many updated references, and also they can consider the following papers:

3. The methodology and data section describes the model adequately and well; and also points out the advantages of the model.

4. In the empirical results section, the pre-estimation tests are performed well, and the superiority of each of them over the other tests is described. The model results are interesting; however, table 6 reports the JHansen and its $p$-value, but the authors did not explain it in the text. Moreover, the data sub-section explained which countries are related to every panel, and it is repeatedly mentioned in the empirical results section. For example, after table 5, “In countries with weak and medium quality of institutions attributed to panels $A$ and $B, \ldots . . "$.

5. Finally, the conclusion section and abstract are well written, and interesting policies are proposed. 